\section{Flare of a Cryopyrin-associated Periodic Syndrome Following Vaccination with Neisseria Meningitidis Polysaccharides}

\section{To the Editor:}

We report the case of a 6-year-old boy with the clinical diagnosis of cryopyrin-associated periodic syndrome (CAPS) ${ }^{1}$. The patient's symptoms had started at 3 years of age and consisted of recurrent cold-induced urticarial rashes in association with arthralgia, myalgia, malaise, abdominal pain, headaches, and fever. The symptoms were classified as a mild to moderate CAPS phenotype (in between Muckle Wells-Syndrome and familial cold autoinflammatory syndrome). The family history was negative for autoinflammatory diseases. In 2016, the boy developed a severe systemic inflammatory episode, shortly after the injection of a meningococcal polysaccharide conjugate vaccine (Nimenrix). Prior vaccinations against influenza, tetanus, diphtheria, polio, rotavirus, and hepatitis A had been uneventful, although pneumococcal vaccinations (Prevenar) in 2008 and 2009 were each associated with high fever, commencing within $24 \mathrm{~h}$ and lasting for several days.

At the time of meningococcal vaccination, the patient was in full remission for several months and had no CAPS-related symptoms. Prior to the scheduled therapy with an anti-interleukin 1 (IL-1) agent, the pretreatment vaccine was administered for travel purposes according to current recommendations ${ }^{2}$. A few hours after the meningococcal vaccination (Nimenrix), the boy developed a painful, indurated, red swelling at the injection site that spread rapidly to involve the entire upper arm (Figure 1A). Systemic symptoms were noted on the subsequent day and included fever up to $39.8^{\circ} \mathrm{C}$, fatigue, and nausea. By the third day, the local injection-site reaction had vanished. The high fever persisted, however, and the patient developed symptoms consistent with a CAPS flare, including an urticarial rash that was similar to the child's previous CAPS rash (Figure 1B). The patient also developed further systemic symptoms (headache, abdominal pain, diarrhea, and myalgia). The rash, along with the other symptoms, resolved spontaneously by Day 6 . We were unable to obtain bloodwork following the vaccination. Ethics board approval was not required in accordance with the policy of the authors' institutions, and patient consent was obtained according to the Declaration of Helsinki.

This case is virtually identical to frequent severe inflammatory episodes that have been described after pneumococcal vaccination in patients with CAPS $^{3,4}$ and Behçet disease ${ }^{5}$. In both autoinflammatory conditions, the vaccine had also elicited signs of reactivation of the underlying disease. In a 6-month period following the administration of meningococcal polysaccharide conjugate vaccine (Nimenrix), the incidence of urticaria was reported as $0.3 \%$ in the general population, and the fever incidence as $8.4 \%{ }^{6}$. The authors, however, believe that the combination of symptoms, their sudden onset and severity, and the observation of similar febrile episodes after prior pneumococcal vaccinations are atypical for adverse reactions seen in the general population, and point to a role of the NLRP3 inflammasome in its pathogenesis.

The meningococcal vaccine administered (Nimenrix) is a quadrivalent conjugate vaccine that contains capsular polysaccharides of meningococcal serogroups A, C, W135, and Y covalently linked to tetanus toxoid as a carrier protein ${ }^{7}$. Whereas systemic inflammatory events (headache, fatigue, gastrointestinal symptoms, and fever) are not uncommon after vaccination with meningococcal conjugates, our patient also developed an urticarial rash, which is consistent with CAPS reactivation.

Meningococcal vaccines contain neither adjuvants nor preservatives but do contain polysaccharides from encapsulated bacteria, which, like pneumococcal polysaccharides, are potent stimulators of Toll-like receptors (TLR) 2 and TLR4, and trigger the NLRP3 inflammasome to synthesize active IL- $1 \beta^{8,9,10}$. Enhanced TLR activation by vaccine components in patients prone to inflammasome hyperactivation as a result of underlying NLRP3 mutations can explain the kinetics, severity, and the systemic feature of the
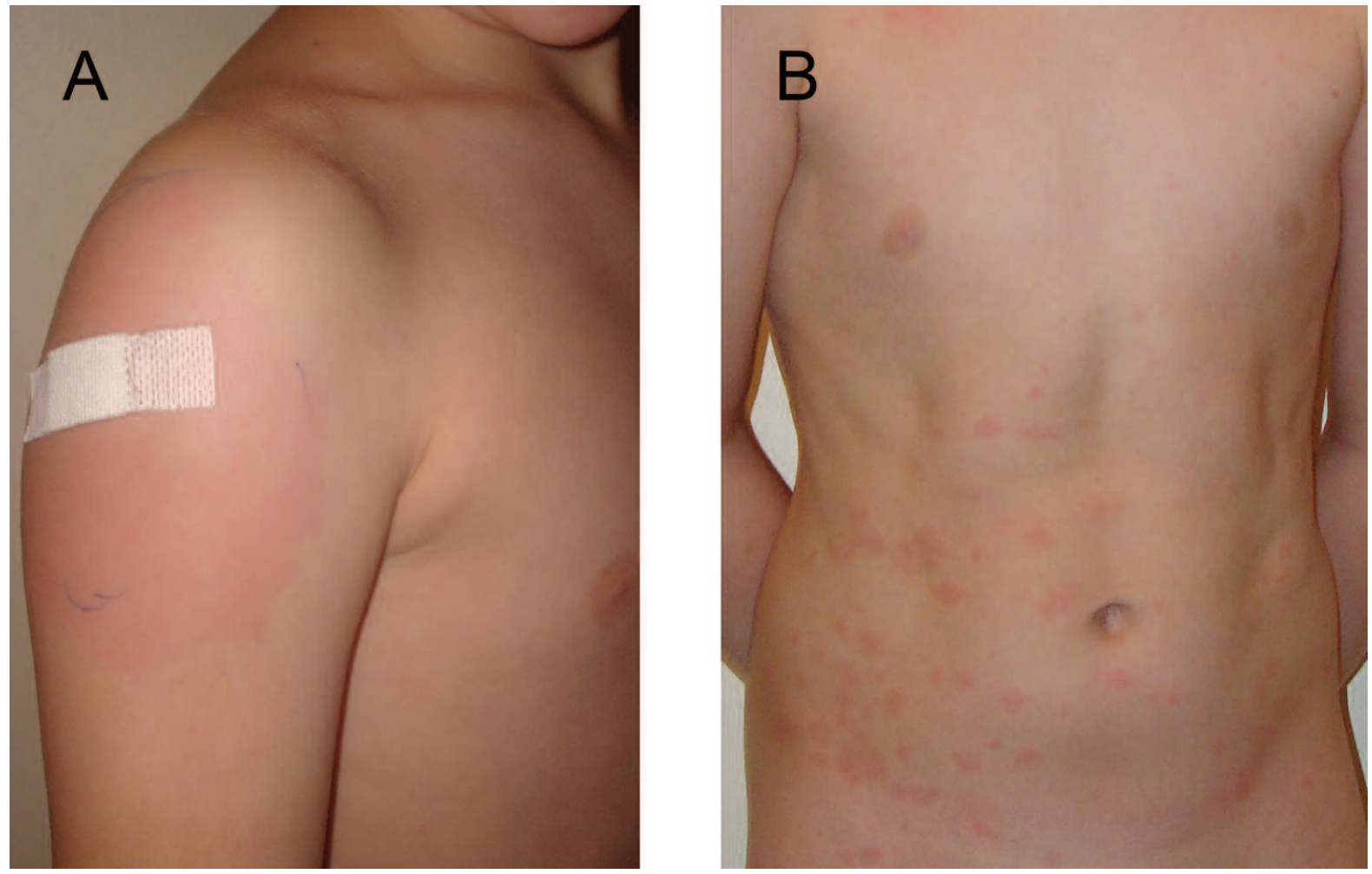

Figure 1. A. Local inflammation in a 6-year-old boy with CAPS at the injection site, 1 day after administration of meningococcal conjugate vaccine. B. Urticarial rash as a sign of systemic CAPS flare (Day 3 after vaccination). CAPS: cryopyrin-associated periodic syndrome.

Personal non-commercial use only. The Journal of Rheumatology Copyright @ 2018 . All rights reserved. 
vaccine reaction in this patient with CAPS. It is unlikely that tetanus toxoid as a covalent carrier protein in the administered meningococcal vaccine had triggered the inflammatory episode, because patients with CAPS were shown to tolerate tetanus and other vaccines well ${ }^{3}$.

To our knowledge, this is the first case of a patient in whom meningococcal vaccination elicited a severe local and systemic inflammatory response, as well as a flare of the underlying autoinflammatory syndrome. As the current vaccination guidelines currently recommend such vaccinations in patients scheduled to receive immunosuppressive medications ${ }^{2}$, additional cases will likely ensue. Research is needed to characterize the incidences of the reactions and adequately balance risks and benefits of meningococcal and other vaccinations in CAPS and other autoinflammatory conditions. The authors no longer administer meningococcal and pneumococcal vaccines to patients with CAPS if either the patients themselves, or other affected family members, had prior reactions to pneumococcal or meningococcal vaccines. The authors continue vaccinating patients with CAPS if the patients themselves and their family members have no prior vaccination reaction to these vaccines.

ULRICH A. WALKER, MD, Unispital Basel, Department of Rheumatology, Basel, Switzerland; SEVERINE LUETKE VOLKSBECK, MD, Private Practice, Bonn; JASMIN KUEMMERLE-DESCHNER, MD, Division of Rheumatology, Department of Pediatrics, University Hospital Tübingen, Tübingen, Germany. Address correspondence to Prof. Dr. U.A. Walker, Unispital Basel, Department of Rheumatology, Basel, Switzerland. E-mail: Ulrich.walker@usb.ch

\section{REFERENCES}

1. Kuemmerle-Deschner JB, Ozen S, Tyrrell PN, Kone-Paut I, Goldbach-Mansky R, Lachmann H, et al. Diagnostic criteria for cryopyrin-associated periodic syndrome (CAPS). Ann Rheum Dis 2017;76:942-7.

2. van Assen S, Agmon-Levin N, Elkayam O, Cervera R, Doran MF, Dougados $\mathrm{M}$, et al. EULAR recommendations for vaccination in adult patients with autoimmune inflammatory rheumatic diseases. Ann Rheum Dis 2011;70:414-22.

3. Jaeger VK, Hoffman HM, van der Poll T, Tilson H, Seibert J, Speziale A, et al. Safety of vaccinations in patients with cryopyrin-associated periodic syndromes: a prospective registry based study. Rheumatology 2017;56:1484-91.

4. Walker UA, Hoffman HM, Williams R, Kuemmerle-Deschner J, Hawkins PN. Brief report: Severe inflammation following vaccination against Streptococcus pneumoniae in patients with cryopyrin-associated periodic syndromes. Arthritis Rheumatol 2016;68:516-20.

5. Hugle T, Bircher A, Walker UA. Streptococcal hypersensitivity reloaded: severe inflammatory syndrome in Behcet's disease following 23-valent polysaccharide Streptococcus pneumoniae vaccine. Rheumatology 2012;51:761-2.

6. European Medicines Agency. Assessment report: Nimenrix. [Internet. Accessed February 21, 2018.] Available from: www.ema.europa.eu/docs/en_GB/document_library/ EPAR_Public_assessment_report/human/002226/WC500127664.pdf

7. Croxtall JD, Dhillon S. Meningococcal quadrivalent (serogroups A, $\mathrm{C}, \mathrm{W} 135$ and $\mathrm{Y}$ ) tetanus toxoid conjugate vaccine (Nimenrix). Drugs 2012;72:2407-30.

8. Zughaier SM. Neisseria meningitidis capsular polysaccharides induce inflammatory responses via TLR2 and TLR4-MD-2. J Leukoc Biol 2011;89:469-80.

9. van der Poll T, Opal SM. Pathogenesis, treatment, and prevention of pneumococcal pneumonia. Lancet 2009;374:1543-56.

10. Sen G, Khan AQ, Chen Q, Snapper CM. In vivo humoral immune responses to isolated pneumococcal polysaccharides are dependent on the presence of associated TLR ligands. J Immunol 2005;175:3084-91.

J Rheumatol 2018;45:6; doi:10.3899/jrheum.171037 